\title{
A Qualitative Analysis of Provider Notes of Atopic Dermatitis-Related Visits Using Natural Language Processing Methods
}

\author{
Evangeline J. Pierce (D) · Natalie N. Boytsov · Joe J. Vasey • \\ Theresa C. Sudaria · Xiong Liu $\cdot$ Kevin W. Lavelle $\cdot$ Alina N. Bogdanov • \\ Orin M. Goldblum
}

Received: April 9, 2021 / Accepted: May 13, 2021 / Published online: May 30, 2021

(C) The Author(s) 2021

\section{ABSTRACT}

Introduction: Real-world disease management of atopic dermatitis (AD) is hampered by a lack of consistency between providers that treat $\mathrm{AD}$ regarding assessment of severity, disease activity, and quality of life. Variability and inconsistency in documentation makes it difficult to understand the impact of AD. This study summarizes AD-related symptoms and concerns captured in unstructured qualitative provider notes by healthcare providers during visits with patients with AD.

Methods: Provider notes were obtained for patients with $\mathrm{AD}(n=133,025)$ from a USAbased ambulatory electronic health records system. The sample included both children $(n=69,551)$ and adults at least 18 years of age ( $n=63,474)$ receiving treatment from a variety of specialties including primary care, dermatology, and allergy/immunology. Key skin-related words were identified from a review of a sample of notes and natural language processing (NLP) was applied to determine the frequency of the keywords and bigram patterns.

Results: Provider notes largely focused on symptoms (primarily itch) and symptom relief rather than the impact of $\mathrm{AD}$ on work or lifestyle. Despite the known relationship between itch and skin pain, neuralgia was not widely documented. Compared to primary care providers, dermatologists' and allergist/immunologists' notes had more documentation of symptom-related issues. Personal and work/life burden issues were not widely documented regardless of specialty.

Conclusion: The topics documented in case notes by healthcare providers about their patients with $\mathrm{AD}$ focus largely on symptoms and, to a lesser extent, treatment, but do not reflect the burden of $\mathrm{AD}$ on patients' lives. This finding highlights a potential care gap that warrants further investigation.

Keywords: Atopic dermatitis; Provider notes; Natural language processing

E. J. Pierce $(\bowtie) \cdot$ N. N. Boytsov $\cdot$ X. Liu ·

O. M. Goldblum

Lilly Corporate Center, Eli Lilly and Company, 893

Delaware St, Indianapolis, IN 46285, USA

e-mail: evangeline.pierce@lilly.com

J. J. Vasey · T. C. Sudaria · K. W. Lavelle ·

A. N. Bogdanov

Veradigm Health, San Francisco, CA, USA 


\section{Key Summary Points}

Healthcare providers' case notes for patients with $\mathrm{AD}$ are largely concerned with symptoms and treatment.

Notes of dermatologists and allergist/ immunologists had more documentation of symptom-related issues than those of primary care providers.

The burden of $\mathrm{AD}$ on daily functioning and quality of life are not generally documented in provider notes regardless of provider specialty.

\section{DIGITAL FEATURES}

This article is published with digital features, including a summary slide, to facilitate understanding of the article. To view digital features for this article go to https://doi.org/10.6084/ m9.figshare.14579118.

\section{INTRODUCTION}

Atopic dermatitis (AD) is a chronic, relapsing, and inflammatory skin disease commonly associated with other atopic manifestations such as food allergy, allergic rhinitis, and asthma $[1,2]$. It is a common skin condition, prevalent in children (12.9\% in the USA) and adults (7.2-10.2\%) [3]. AD can have a longlasting and significant impact on quality of life (QoL), particularly with regard to psychological and physical well-being and social functioning $[4,5]$. Adult patients with $\mathrm{AD}$ report greater dissatisfaction with life and poorer overall mental and physical health compared with those without $\mathrm{AD}$ [6]. The general effect of $\mathrm{AD}$ on adult QoL is reported to be greater than other skin conditions such as psoriasis or urticaria, although the specifics of the impairment profiles differ between the conditions [7].
$\mathrm{AD}$ is characterized by a wide range of dermatological manifestations. Although there are several validated sets of diagnostic criteria, there is disagreement about its definition $[8,9]$. As a result, the variety of different definitions and assessments that have been utilized in $\mathrm{AD}$ studies may compromise the generalizability of results and conclusions across different studies [9]. An international effort to develop a harmonized set of measures (the Harmonizing Outcome Measures for Eczema initiative) has called attention to the need for minimum requirements for core definitions and features (clinical signs, symptoms, long-term control, QoL) [10].

An understanding of real-world disease management is hampered by a lack of consistency between providers in various specialties that treat $\mathrm{AD}$ in terms of their assessment of severity, disease activity, and impact on QoL. Furthermore, clinical studies require rigorous, valid, and widely accepted diagnostic criteria for reliable and reproducible research. These kinds of assessments are often not used in clinical ambulatory settings. A move towards standardization of AD-related nomenclature has the potential to improve generalizability of clinical trials and, by extension, epidemiological studies.

The value of provider notes was suggested in recent work that utilized structured data and unstructured provider notes to assist in identifying patients with AD [11]. When combined with structured data, disease concepts (signs, symptoms, activity) identified through natural language processing (NLP) of provider notes were found to significantly improve the performance of an $\mathrm{AD}$ disease identification algorithm based on structured data alone.

In this study, NLP-derived information from notes was used to summarize disease-related symptoms captured by healthcare providers (HCP) during visits with patients with AD. This, coupled with structured data from the electronic health record (EHR), offers the potential for a broader understanding of $\mathrm{AD}$ management and treatment. 


\section{METHODS}

\section{Study Design and Data Sources}

This research is a retrospective, observational cohort study of adults and children with a diagnosis of AD. Structured data and unstructured provider notes were sourced from the Practice Fusion EHR system (Practice Fusion, San Francisco, CA, USA), a platform used throughout the USA, that records clinical data for approximately $6 \%$ of all ambulatory care (both primary care and specialist practices) in the USA [12]. It is comparable to the overall USA population in terms of age, gender, and geographic location, as described in the National Ambulatory Medical Care Survey 2014 [13]. Practices using the EHR tend to be small (five or fewer HCPs) and independent. The structured patient data include demographics, diagnoses and comorbidities, laboratory test orders and results, and prescriptions written by the treating physician as well as documentation of those written by other HCPs. The unstructured provider notes describe HCPs' observations and assessments of patients' condition, progress, concerns, and management and treatment plans. The data are contained in a de-identified, Health Insurance Portability and Accountability Act (HIPAA)-compliant research-certified database covering the years 2014-2020. The retrospective EHR dataset used for this study is HIPAA compliant and statistically certified for retrospective research without institutional review board (IRB) review.

\section{Patients' Inclusion Criteria and Providers' Specialty}

Patients regardless of age were included in the study cohort if they had a coded diagnosis of $\mathrm{AD}$ (Table 1) in the EHR system prior to the

Table 1 AD diagnosis codes and AD-associated medications

\begin{tabular}{lll}
\hline Diagnosis code & Condition & AD-associated medications \\
\hline ICD-9-CM & & Conventional oral immunosuppressants \\
691 & Atopic dermatitis and related conditions & IgE inhibitors \\
691.8 & Other atopic dermatitis and related conditions & IL-4/IL-13 inhibitors \\
ICD-10-CM & & Immunoglobulin \\
L20 & Atopic dermatitis & JAK inhibitors \\
L20.0 & Besnier's prurigo & Oral antihistamines \\
L20.8 & Other atopic dermatitis & Oral corticosteroids \\
L20.81 & Atopic neurodermatitis & PDE4 inhibitors \\
L20.82 & Flexural eczema & Retinoids \\
L20.83 & Infantile (acute or chronic) eczema & Topical antihistamines \\
L20.84 & Intrinsic (allergic) eczema & Topical calcineurin Inhibitors \\
L20.89 & Other atopic dermatitis & Topical corticosteroids \\
L20.9 & Atopic dermatitis & Topical PDE4 inhibitors \\
& & Vitamin D $_{3}$ analogues \\
& & Interferon gamma \\
\hline
\end{tabular}

IgE immunoglobulin E, Il interleukin, PDE4 phosphodiesterase 4, ICD-9/10-CM International Classification of Diseases 9th/10th Edition, Clinical Modification 
study observation period and an AD-related office visit during the 30-month period ending in August 2019. The timeframe reflects the most current data available when the study was initiated. Within the study period, a patient's earliest visit date for $\mathrm{AD}$ was designated as the index visit. Patients were classified as children (age less than 18 years) or adults (age 18 years or more) based on their age at the index visit.

Providers were classified by specialty as allergist/immunologists, dermatologists, primary care (adult), primary care (pediatrics), and other. Specialties in the "other" category who provided AD-related care included podiatrists, naturopaths, nurse practitioners, and providers not reporting a specialty.

\section{Data Processing}

Demographic, clinical, and pharmacotherapy data were obtained from the structured EHR. Data were used as recorded in the chart by HCPs-imputation was not performed for data points (race, ethnicity, location, insurance type) that were not recorded or were otherwise missing. AD-associated comorbid conditions were identified by ICD-9-CM and ICD-10-CM diagnosis coding. Pharmacotherapy as of the index visit was determined from prescription records of scripts written by the HCP electronically or on paper, written by HCPs outside the practice, or documented as something the patient took historically. From the perspective of the EHR, a prescription record represents an intent to treat, not the actual fulfillment or use of the prescribed medication. AD-associated topical and systemic (oral and injectable) medications (Table 1) as of the index visit were identified, coded, and classified using the National Drug Code, 11-digit standard (NDC-11).

The process of developing a set of topics from notes for patients with $\mathrm{AD}$ began with a review of skin-related terms and phrases from a sample of 100 randomly selected notes associated with an $\mathrm{AD}$ visit. Notes were sampled from multiple specialties and patients of varying age and gender. Word frequencies were generated from these notes and reviewed to identify and focus on skin-related terms of interest. From this word frequency list a subset of nine key terms were identified for further investigation, categorized into three dimensions: disease-related (atopic) treatment-related (systemic, topical), symptom-related (itch, sleep, neuralgia, pain), and personal-related (life, work).

Notes were tokenized to break running text into sentences and individual words which were lemmatized to reduce them to their base form. Lemmatization allows different forms of the same word to be grouped and treated similarly. An example of lemmatization is "itch", which is the base form for "itches" and "itchy". Lemmatization operates at the word level and is not intended to make inferences about synonyms or to develop overarching ontological concepts. For every sentence in which one of the nine key lemmatized words occurred, other skin-relevant words were identified through the word frequency analysis described above. Word pairs (bigrams) were formed between the keyword and each skin relevant word, indicating the adjacency of the two terms. Bigram components are not necessarily immediately adjacent to one another but may be separated by intermediate connecting words.

For each of the nine keywords a frequency analysis was conducted to determine the 25 most common bigrams pairs of those keywords with other skin-related words. Circular network graphs show the relationship between a nodal keyword term (e.g., "itch") and adjacent words in sentences containing the keyword. Similar to word clouds, they graphically represent the cooccurrence of other terms with the keyword and strength of the relationship between the keyword and related words. Starting at the keyword and traveling counterclockwise around the graph, words closest to the keyword represent higher frequency bigrams or phrases, while words farther away are in less frequently occurring phrases. In some cases, bigrams occur in sentences that do not involve the keyword. These are indicated in the network graphs by the absence of a connecting line between the keyword and the non-adjacent word. Distance from the keyword in a counterclockwise direction is indicative of the frequency of the bigram. 


\section{RESULTS}

The sample of 133,025 patients with $\mathrm{AD}$ (Table 2) consisted of 69,551 (52.3\%) children (age less than 18 years) and 63,474 (47.7\%) adults (age 18 years or older). The average age was 5.9 years $(\mathrm{SD}=5.0)$ for children and 49.9 years $(\mathrm{SD}=19.3)$ for adults. Overall, slightly over half of the patients were female $(55.7 \%)$. To the extent that race/ethnicity is recorded in the EHR, the majority were white and non-Hispanic. Primary care and pediatric practices accounted for the most patients (39.9\% and $35.7 \%$ of all AD cases, respectively). Allergists and immunologists provided care for $11.2 \%$ of patients with $\mathrm{AD}$, and dermatologists accounted for $7.2 \%$. This contrasts with the nearly 2:1 ratio of dermatologists to allergists/ immunologists in the USA [14] and is reflective of the composition of the user base of the EHR.

Records were examined for the prevalence of six AD-associated comorbidities (Table 3). Regardless of age, the most common documented comorbidity was allergic rhinitis (23.9\%). The condition was slightly more common among children (26.0\%) than adults (21.5\%). Asthma was also widely diagnosed $(15.2 \%)$, at similar rates among children (15.9\%) and adults (14.4\%). Rhinosinusitis was slightly more prevalent among adults (4.0\%) than children $(2.1 \%)$, while food allergies were more often seen in children $(6.7 \%$ vs. $1.7 \%$ for adults).

The use of AD-related medications as of the index visit is reported in Table 3. Topical corticosteroids were the most widely prescribed (58.6\% of the sample), with $60.8 \%$ of children and $56.1 \%$ of adults having a prescription. Oral antihistamines $(20.9 \%$ of the sample) and oral corticosteroids (19.5\%) were the next most widely prescribed medications. Oral corticosteroids were more commonly prescribed for adults $(23.1 \%)$ than for children $(16.2 \%)$. Oral antihistamine prescriptions were found in similar proportions of adults and children $(20.5 \%$ and $21.3 \%$, respectively). Injectable biologics (IgE or IL-4/IL-13 inhibitors) and conventional oral immunosuppressants (azathioprine, cyclosporine, methotrexate, mycophenolate
Table 2 Patient characteristics

\begin{tabular}{|c|c|c|}
\hline Characteristic & Number & Percentage \\
\hline \multicolumn{3}{|l|}{ Age at index } \\
\hline $\begin{array}{l}\text { Total sample mean } 28.2 \\
(\mathrm{SD}=27.4)\end{array}$ & 133,025 & 100.0 \\
\hline $\begin{array}{l}\text { Children }(<18) \text { mean } 5.9 \\
(\mathrm{SD}=5.0)\end{array}$ & 69,551 & 52.3 \\
\hline $\begin{array}{l}\text { Adults }(\geq 18) \text { mean } 49.9 \\
(S D=19.3)\end{array}$ & 63,474 & 47.7 \\
\hline \multicolumn{3}{|l|}{ Gender } \\
\hline Female & 74,147 & 55.7 \\
\hline \multicolumn{3}{|l|}{ Race } \\
\hline Black/African American & 11,857 & 8.9 \\
\hline White & 29,675 & 22.3 \\
\hline Race other than Black or White & 12,351 & 9.3 \\
\hline Undocumented & 79,142 & 59.5 \\
\hline Hispanic ethnicity & 19,013 & 14.3 \\
\hline \multicolumn{3}{|l|}{ Census region } \\
\hline Midwest & 17,533 & 13.2 \\
\hline Northeast & 21,812 & 16.4 \\
\hline South & 51,465 & 38.7 \\
\hline West & 33,765 & 25.4 \\
\hline Undocumented & 8450 & 6.4 \\
\hline \multicolumn{3}{|l|}{ Insurance } \\
\hline Commercial & 43,526 & 32.7 \\
\hline Medicare/Medicaid & 15,502 & 11.7 \\
\hline Self-pay & 812 & 0.6 \\
\hline Undocumented & 73,185 & 55.0 \\
\hline \multicolumn{3}{|l|}{ Specialty } \\
\hline Allergy and Immunology & 14,833 & 11.2 \\
\hline Derm-specific & 9534 & 7.2 \\
\hline PCP & 53,130 & 39.9 \\
\hline Pediatrics & 47,427 & 35.7 \\
\hline Other & 8101 & 6.1 \\
\hline
\end{tabular}


Table 3 Patient clinical and treatment characteristics

\begin{tabular}{|c|c|c|c|c|c|c|}
\hline & \multicolumn{2}{|l|}{ Total } & \multicolumn{2}{|c|}{ Children $(<18)$} & \multicolumn{2}{|c|}{ Adults $(18+)$} \\
\hline & $n$ & $\%$ & $n$ & $\%$ & $n$ & $\%$ \\
\hline \multicolumn{7}{|l|}{ Comorbid condition } \\
\hline Asthma & 20,211 & 15.19 & 11,049 & 15.89 & 9162 & 14.43 \\
\hline Allergic rhinitis & 31,726 & 23.85 & 18,110 & 26.04 & 13,616 & 21.45 \\
\hline Rhinosinusitis & 3992 & 3.00 & 1456 & 2.09 & 2536 & 4.00 \\
\hline Food allergy & 5741 & 4.32 & 4662 & 6.70 & 1079 & 1.70 \\
\hline Chronic urticaria & 0 & 0.00 & 0 & 0.00 & 0 & 0.00 \\
\hline Eosinophilic esophagitis & 74 & 0.06 & 38 & 0.05 & 36 & 0.06 \\
\hline \multicolumn{7}{|l|}{ Medication class } \\
\hline Conventional oral immunosuppressants & 678 & 0.51 & 49 & 0.07 & 629 & 0.99 \\
\hline IgE inhibitors & 117 & 0.09 & 18 & 0.03 & 99 & 0.16 \\
\hline IL-4/IL-13 inhibitors & 219 & 0.16 & 12 & 0.02 & 207 & 0.33 \\
\hline Immune globulin & 16 & 0.01 & 5 & 0.01 & 11 & 0.02 \\
\hline Interferon gamma & 0 & 0.00 & 0 & 0.00 & 0 & 0.00 \\
\hline JAK inhibitors & 20 & 0.02 & 0 & 0.00 & 20 & 0.03 \\
\hline Oral antihistamines & 27,828 & 20.92 & 14,819 & 21.31 & 13,009 & 20.50 \\
\hline Oral corticosteroids & 25,972 & 19.52 & 11,300 & 16.25 & 14,672 & 23.11 \\
\hline PDE4 Inhibitors & 45 & 0.03 & 0 & 0.00 & 45 & 0.07 \\
\hline Retinoids & 68 & 0.05 & 8 & 0.01 & 60 & 0.09 \\
\hline Topical antihistamines & 766 & 0.58 & 124 & 0.18 & 642 & 1.01 \\
\hline Topical calcineurin Inhibitors & 2,422 & 1.82 & 1086 & 1.56 & 1336 & 2.10 \\
\hline Topical corticosteroids & 77,912 & 58.57 & 42,289 & 60.80 & 35,623 & 56.12 \\
\hline Topical PDE4 inhibitors & 2809 & 2.11 & 1231 & 1.77 & 1578 & 2.49 \\
\hline Vitamin $\mathrm{D}_{3}$ analogues & 191 & 0.14 & 14 & 0.02 & 177 & 0.28 \\
\hline Patients with 1 drug class & 52,955 & 39.81 & 24,910 & 35.82 & 28,045 & 44.18 \\
\hline Patients with 2 drug classes & 27,080 & 20.36 & 13,226 & 19.02 & 13,854 & 21.83 \\
\hline Patients with $3+$ drug classes & 10,245 & 7.70 & 5316 & 7.64 & 4929 & 7.77 \\
\hline Patients with no medications & 42,745 & 32.13 & 26,099 & 37.52 & 16,646 & 26.22 \\
\hline
\end{tabular}

$I g E$ immunoglobulin E, $I l$ interleukin, PDE4 phosphodiesterase 4

mofetil, tacrolimus) were prescribed to less than $1 \%$ of patients, generally adults. No patients were on interferon gamma treatment.
Over a third of the patient sample had a prescription from a single class of drugs $(39.8 \%)$. Less than $10 \%$ of the sample had prescriptions from three or more classes. Patients 
Table 4 Patient clinical and treatment characteristics

\begin{tabular}{|c|c|c|c|c|c|c|c|c|c|c|}
\hline & \multicolumn{2}{|c|}{$\begin{array}{l}\text { Allergy/ } \\
\text { immunology }\end{array}$} & \multicolumn{2}{|c|}{ Dermatology } & \multicolumn{2}{|c|}{ Other } & \multicolumn{2}{|c|}{ PCP-adults } & \multicolumn{2}{|c|}{$\begin{array}{l}\text { PCP- } \\
\text { pediatrics }\end{array}$} \\
\hline & $\bar{n}$ & $\%$ & $\bar{n}$ & $\%$ & $\bar{n}$ & $\%$ & $\bar{n}$ & $\%$ & $\bar{n}$ & $\%$ \\
\hline \multicolumn{11}{|l|}{ Comorbid condition } \\
\hline Asthma & 4009 & 27.03 & 379 & 3.98 & 727 & 8.97 & 7905 & 14.88 & 7191 & 15.16 \\
\hline Allergic rhinitis & 8132 & 54.82 & 318 & 3.34 & 832 & 10.27 & 11,812 & 22.23 & 10,632 & 22.42 \\
\hline Rhinosinusitis & 657 & 4.43 & 60 & 0.63 & 147 & 1.81 & 2162 & 4.07 & 966 & 2.04 \\
\hline Food allergy & 3214 & 21.67 & 13 & 0.14 & 96 & 1.19 & 493 & 0.93 & 1925 & 4.06 \\
\hline Chronic urticaria & 0 & 0.00 & 0 & 0.00 & 0 & 0.00 & 0 & 0.00 & 0 & 0.00 \\
\hline Eosinophilic esophagitis & 45 & 0.30 & 1 & 0.01 & 4 & 0.05 & 8 & 0.02 & 16 & 0.03 \\
\hline \multicolumn{11}{|l|}{ Medication class } \\
\hline Conventional oral immunosuppressants & 54 & 0.36 & 174 & 1.83 & 47 & 0.58 & 381 & 0.72 & 22 & 0.05 \\
\hline IgE inhibitors & 68 & 0.46 & 12 & 0.13 & 2 & 0.02 & 32 & 0.06 & 3 & 0.01 \\
\hline IL-4/IL-13 inhibitors & 51 & 0.34 & 75 & 0.79 & 15 & 0.19 & 74 & 0.14 & 4 & 0.01 \\
\hline Immune globulin & 9 & 0.06 & 1 & 0.01 & 1 & 0.01 & 4 & 0.01 & 1 & 0.00 \\
\hline Interferon gamma & 0 & 0.00 & 0 & 0.00 & 0 & 0.00 & 0 & 0.00 & 0 & 0.00 \\
\hline JAK inhibitors & 1 & 0.01 & 4 & 0.04 & 3 & 0.04 & 12 & 0.02 & 0 & 0.00 \\
\hline Oral antihistamines & 4531 & 30.55 & 991 & 10.39 & 927 & 11.44 & 11,873 & 22.35 & 9506 & 20.04 \\
\hline Oral corticosteroids & 2074 & 13.98 & 658 & 6.90 & 1024 & 12.64 & 14,033 & 26.41 & 8183 & 17.25 \\
\hline PDE4 inhibitors & 6 & 0.04 & 12 & 0.13 & 5 & 0.06 & 22 & 0.04 & 0 & 0.00 \\
\hline Retinoids & 2 & 0.01 & 35 & 0.37 & 3 & 0.04 & 26 & 0.05 & 2 & 0.00 \\
\hline Topical antihistamines & 74 & 0.50 & 76 & 0.80 & 41 & 0.51 & 521 & 0.98 & 54 & 0.11 \\
\hline Topical calcineurin inhibitors & 748 & 5.04 & 595 & 6.24 & 54 & 0.67 & 643 & 1.21 & 382 & 0.81 \\
\hline Topical corticosteroids & 7951 & 53.60 & 5774 & 60.56 & 2427 & 29.96 & 31,688 & 59.64 & 30,072 & 63.41 \\
\hline Topical PDE4 inhibitors & 666 & 4.49 & 233 & 2.44 & 69 & 0.85 & 1231 & 2.32 & 610 & 1.29 \\
\hline Vitamin $\mathrm{D}_{3}$ analogues & 5 & 0.03 & 50 & 0.52 & 10 & 0.12 & 118 & 0.22 & 8 & 0.02 \\
\hline Patients with 1 drug class & 4984 & 33.60 & 4325 & 45.36 & 2093 & 25.84 & 21,638 & 40.73 & 19,915 & 41.99 \\
\hline Patients with 2 drug classes & 3212 & 21.65 & 1512 & 15.86 & 830 & 10.25 & 12,240 & 23.04 & 9286 & 19.58 \\
\hline Patients with $3+$ drug classes & 1492 & 10.06 & 391 & 4.10 & 283 & 3.49 & 4690 & 8.83 & 3389 & 7.15 \\
\hline Patients with no medications & 5145 & 34.69 & 3306 & 34.68 & 4895 & 60.42 & 14,562 & 27.41 & 14,837 & 31.28 \\
\hline
\end{tabular}

$I g E$ immunoglobulin E, $I l$ interleukin, $P C P$ primary care provider, $P D E 4$ phosphodiesterase 4 


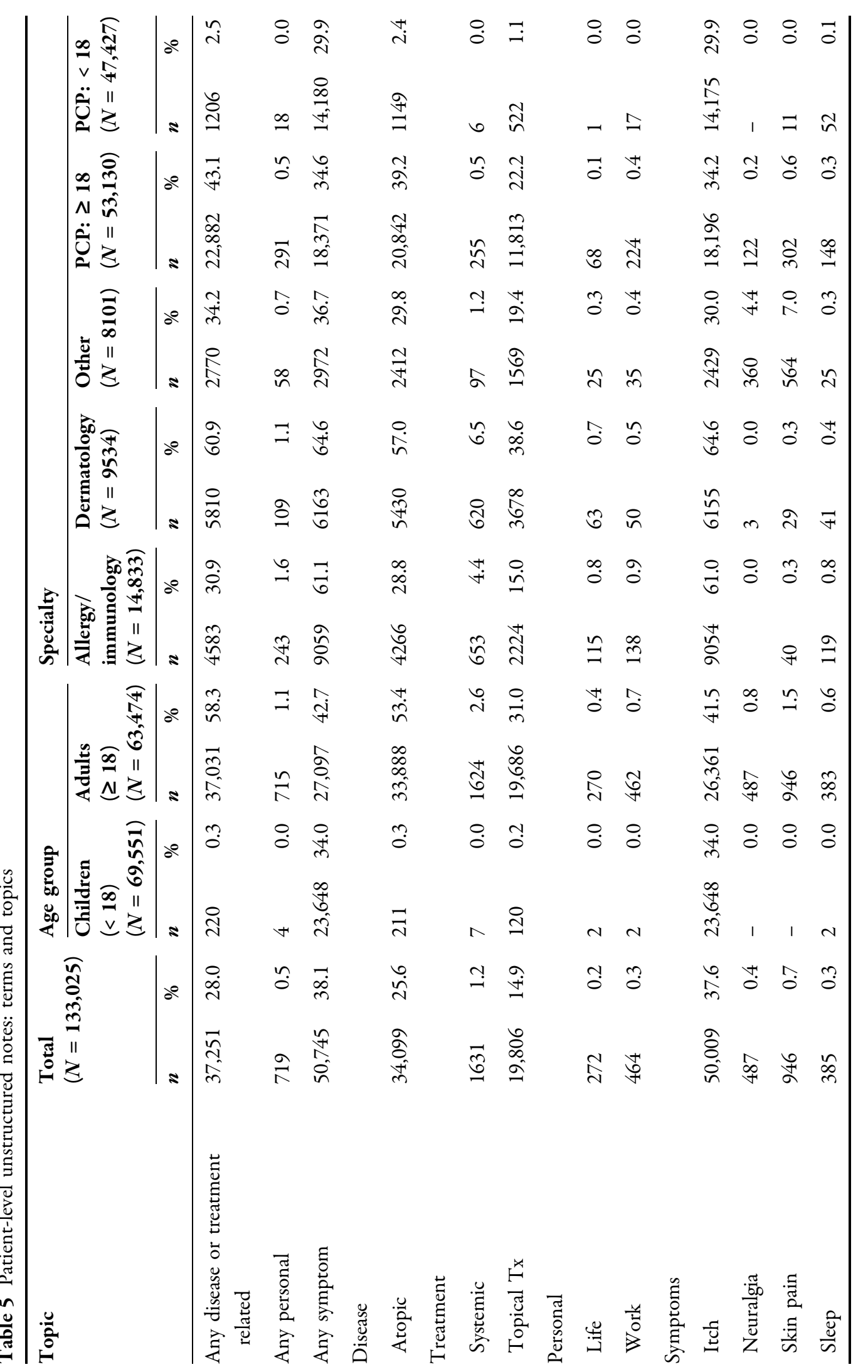




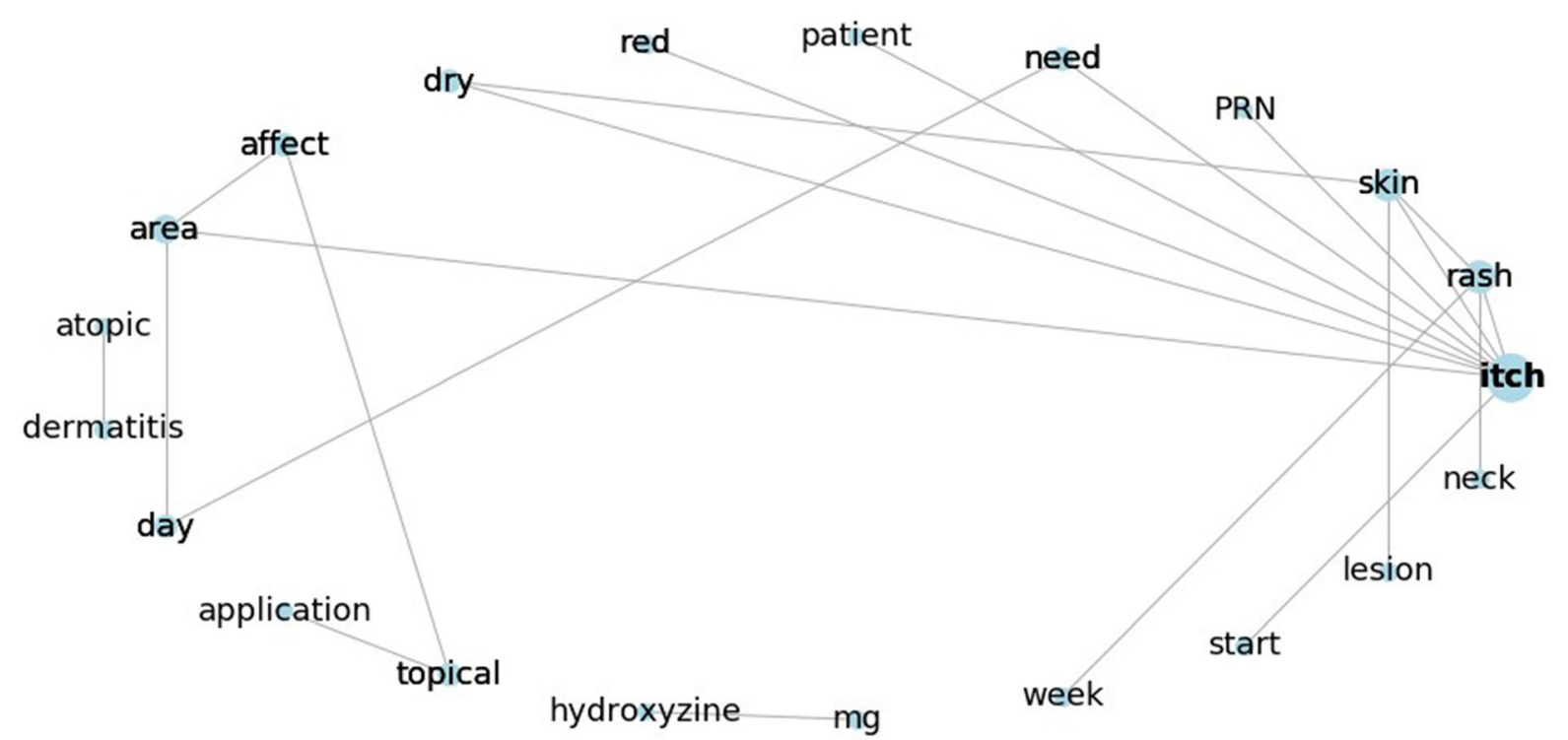

Fig. 1 Bigram network for “itch”. PRN pro re nata (as needed)

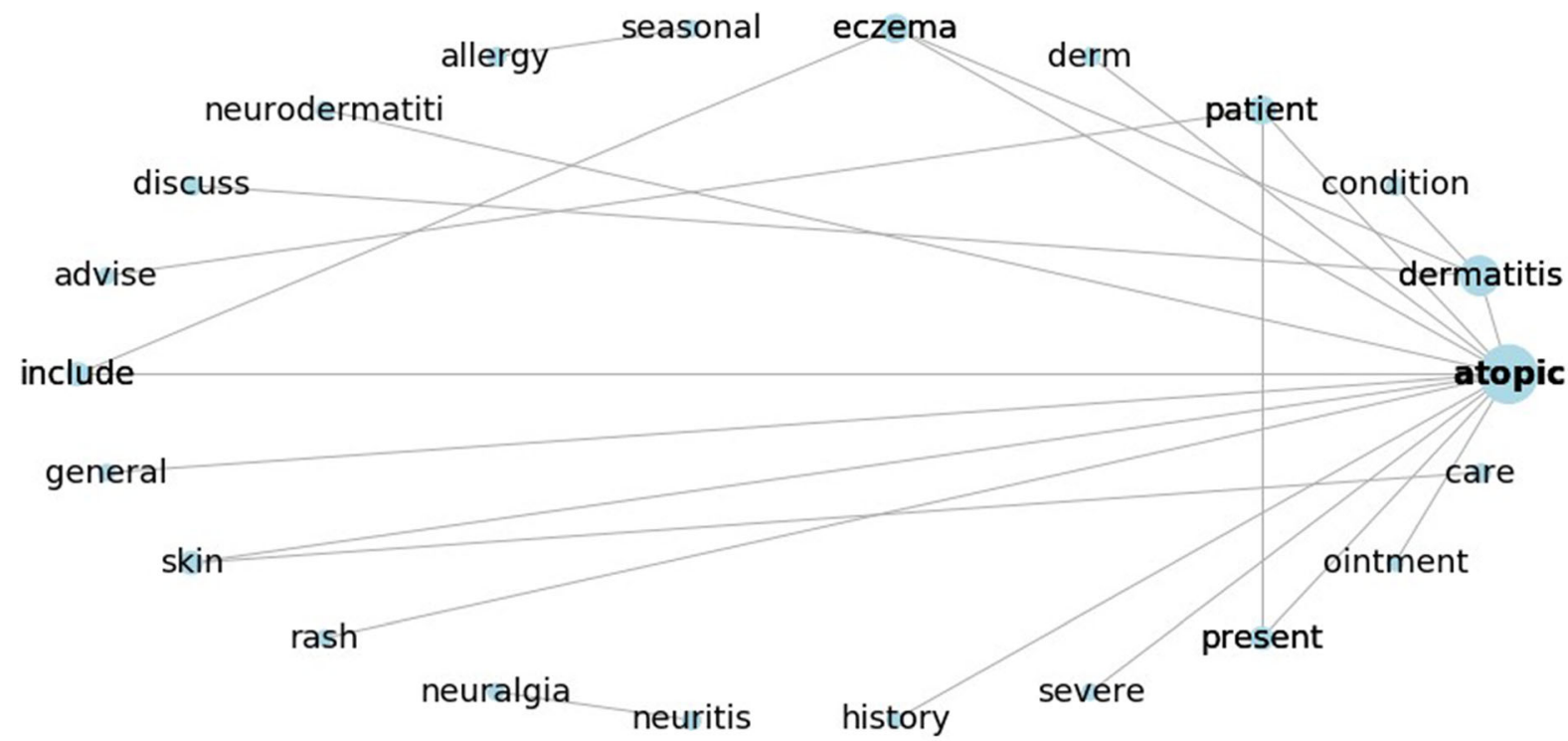

Fig. 2 Bigram network for "atopic"

seeing allergists/immunologists more often had three or more drug classes $(10.1 \%)$ than those seeing dermatologists $(4.1 \%)$, PCP-pediatrics $(7.2 \%)$, or PCP-adults $(8.8 \%)$ (Table 4$)$. Over one-third of children (37.5\%) and a quarter of adults $(26.2 \%)$ had no record of a prescription for an AD-related medication. This included approximately one-third of patients seeing allergists/immunologists (34.7\%), dermatologists (34.7\%), PCP-pediatrics (31.3\%), and PCPadults (27.4\%). Most patients seeing other specialties had no AD-related medications (60.4\%).

Table 5 summarizes the occurrence of disease, treatment, symptom, and work/lifestylerelated terms in provider notes. Disease-related and treatment terms were documented in $28 \%$ 


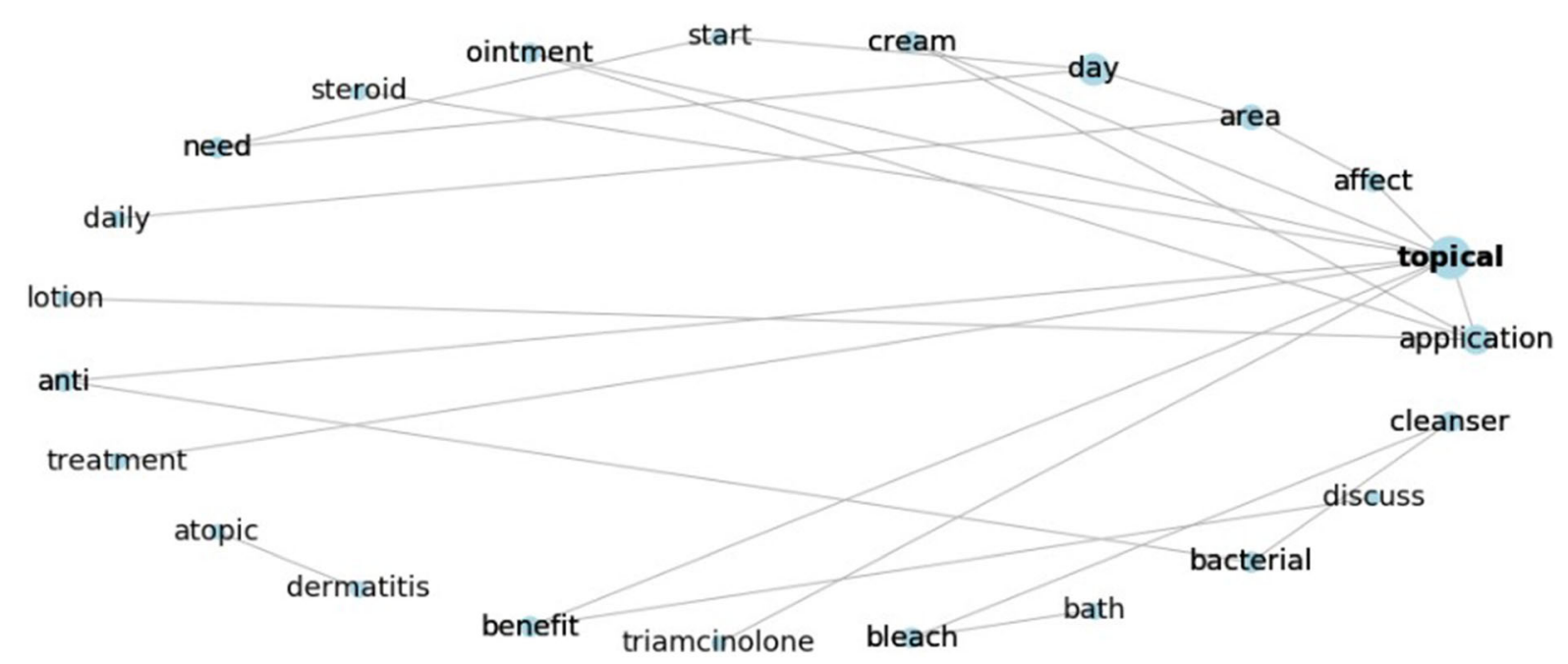

Fig. 3 Bigram network for "topical"

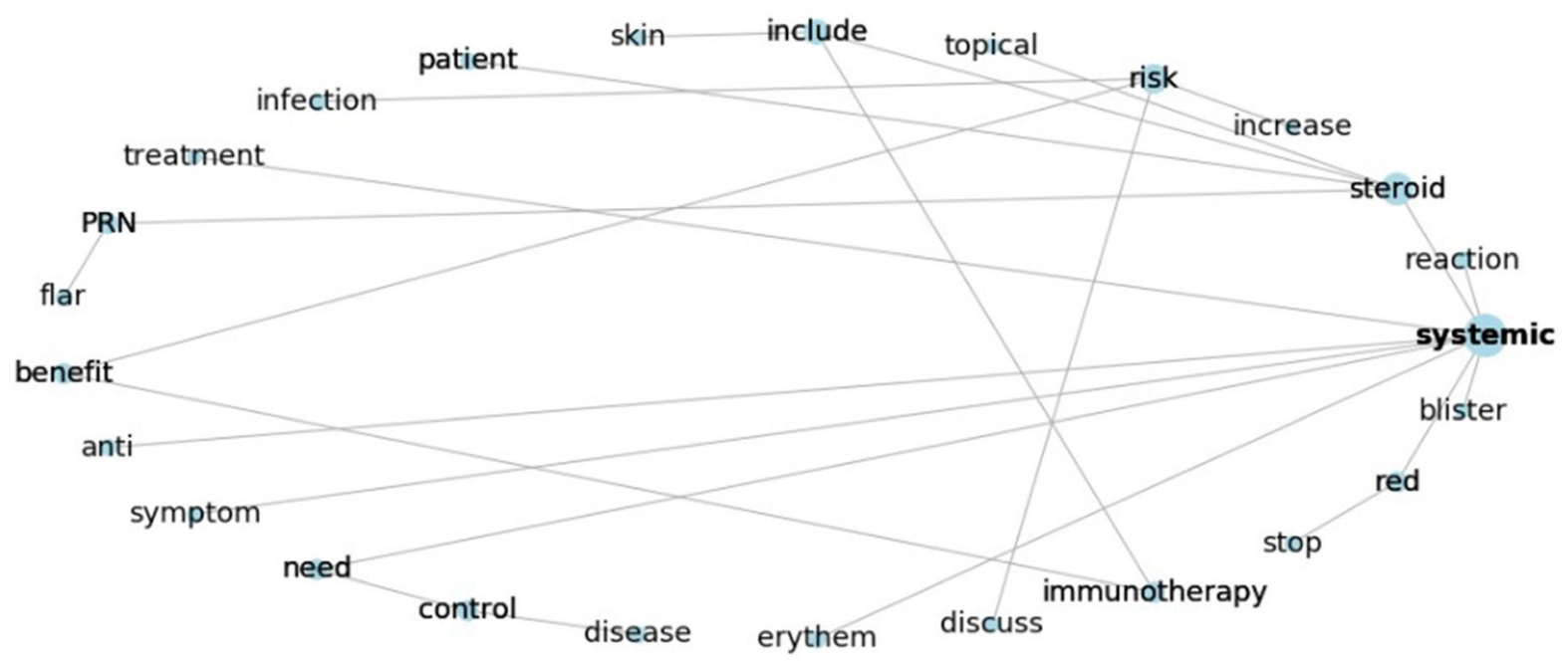

Fig. 4 Bigram network for "neuralgia". PRN pro re nata (as needed)

of patient notes. They were not well documented for children $(0.3 \%)$, but occurred for over half of adult patients (58.3\%). Disease-related and treatment terms were most often mentioned for patients of dermatologists $(60.9 \%)$ and PCPs treating adults $(43.1 \%)$. In contrast, PCPs treating children mentioned them less often (2.5\% of patients). The specific topics most often documented were related to the general atopic nature of the condition (25.6\% overall, $53.4 \%$ for adults). Dermatologists documented these topics for over $50 \%$ of their patients, followed by PCPs with adult patients (39.2\%).

Symptoms (itch, neuralgia, pain, sleep) were the predominant note content $(39.0 \%$ of all patients). Allergists/immunologists and dermatologists provided the most symptom-related information $(61.1 \%$ and $65.3 \%$ of patients, respectively) followed by PCPs (35.3\% of adult patients, $30.0 \%$ of pediatric patients). The most heavily documented term was itch $(37.6 \%$ of all patients, $34 \%$ of children, $41.5 \%$ of adults), mentioned for nearly two-thirds of allergists' immunologists' and dermatologists' patients, 
and for nearly one-third of PCP patients. Neuralgia, skin pain, and sleep disturbances were each mentioned for less than $2 \%$ of patients, regardless of patient age. HCPs from the "other specialties" category, such as those who do not have a specialty noted or podiatrists, naturopaths, or nurse practitioners, tended to document these topics more often (neuralgia $4.4 \%$, pain $7.0 \%$ ). Sleep issues were not often mentioned $(0.3 \%$ of children and $0.6 \%$ of adults $)$ and more often occurred in allergist/immunologist notes $(0.8 \%)$ than in other specialties.

Personal terms related to lifestyle or work were rarely documented ( $0.5 \%$ of all patients). Documentation of the personal aspects of $\mathrm{AD}$ were almost never recorded for children, and life issues were rarely mentioned in adults' notes $(0.4 \%)$. Work-related terms came up slightly more frequently $(0.7 \%$ of adult patients). Allergists/immunologists were somewhat more likely to make notes regarding personal topics (lifestyle $0.8 \%$, work $0.9 \%$ ), followed by dermatologists $(0.7 \%$ and $0.5 \%$, respectively).

Figure 1 presents the bigram analysis for "itch", the most heavily documented symptom assessed (37.6\% of all patients). Sentences dealing with itch focus largely on related symptoms (rash, burn, redness, swelling, congestion, lesions), treatment (hydroxyzine, topical, cream), or more general terms (patient, skin, change, atopic, dermatitis). As suggested by the counterclockwise relative locations of the terms, symptoms were mentioned somewhat more often than treatments. Bigrams occurred in "itch" sentences not involving the keyword-these are indicated by connections between non-nodal terms on the graph and include "skin rash", "dry skin", "dry redness", and "atopic dermatitis".

The atopic characteristics of $\mathrm{AD}$ were the second most widely documented topic $(25.6 \%$ of all patients). Figure 2 bigrams show "atopic" is mentioned most often in relationship with words describing the skin (eczema, dermatitis, skin, rash), severity (severe, mild, acute, improvement), complications (neuralgia, neuritis), or treatment (ointment, topical). Other bigrams occurring in atopic sentences indicate conferences with patients ("patient discussion"), treatments ("topical ointment"), and discomfort ("neuralgia neuritis").

Treatment-related terms are the primary subjects dealt with by the "topical" term (Fig. 3). Words related to this term include ointment, cream, lotion, hydrocortisone, treatment, medication, and bath. Symptoms (rash, itch, stop) are also related to the term, as are several general terms (infect, atopic, dermatitis).

Figure 4 shows bigram relationships having to do with systemic treatment. "Systemic" forms significant bigrams with terms related to management and treatment (reaction, steroid, treatment) as well as terms related to signs and symptoms (symptom, blister, red, erythema). Other bigrams (steroid with topical, include, patient, PRN) reflect a concern with both treatment and its expected benefits (benefit with risk, benefit with immunotherapy).

\section{DISCUSSION}

Structured data provide an objective description of patient characteristics. Demographics, comorbidities, and treatment activities are documented with standard codes over time, providing researchers additional insight into patients diagnosed with AD. Unstructured data from provider notes supplement this material, illustrating specific topics providers and patients are discussing during a visit. This study showed that provider notes largely centered around symptoms (primarily itch) and symptom relief, and not around the impact AD might have on a patient's work or life.

The most documented topics were related to symptoms and disease occurrence, treatment, and characteristics of the disease. The symptom most often mentioned, regardless of provider specialty or patient age, was itch. Itch was relatively well documented, particularly for patients of dermatologists and allergist/immunologists. This reflects finding of other studies which note that itch is the primary defining symptom for $\mathrm{AD}$ [15]. For pediatric patients the focus was almost entirely on symptoms, specifically itch, while for adults, the notes tended to focus on symptoms as well as treatment. 
Severe itch can cause sleep disruption, irritability, and stress and often leads to scratching that may damage the skin, resulting in pain. Distinct from itching, skin pain is an important symptom reported by nearly two-thirds of patients in published research [16]. In provider notes pain and neuralgia were not widely documented, being mentioned for less than $1 \%$ of patients. This finding is in contrast to AD burden and QoL studies that show that skin pain is a common and often severe condition with variable frequency, intensity, and impact on QoL among patients with AD $[16,17]$. Skin pain often leads to sleep disruption and other QoL issues [17]. Sleep disturbances affect between $47 \%$ and $80 \%$ of children and $33-87 \%$ of adults with AD [18]. Next to itch, sleep disturbances are one of the leading factors impacting the QoL of children with AD [19], so it is noteworthy that it is so rarely mentioned in provider notes.

It is also notable that the impact of $\mathrm{AD}$ on QoL, life, and work was rarely documented, regardless of provider specialty or patient age. These topics were found in notes of less than 1\% of patients. Providers might not have enough time during a patient visit to focus beyond disease assessment and treatment considerations. Numerous studies have demonstrated a significant impact of disease activity and severity on QoL [20-22]. AD can have a substantial negative impact on the QoL of children and their families $[4,23]$. The fact that the impact of $\mathrm{AD}$ on patients is generally not captured in the notes suggests that clinicians may not be fully understanding the burden that $\mathrm{AD}$ brings to patients and the role it might play in disease management.

Documentation of disease, personal, or symptomatology issues was greater in dermatologists' notes than for patients in other specialties. Dermatologists documented symptom and broader disease concepts for almost twothirds of their patients. Allergists and immunologists had a similar focus on symptom documentation but recorded less information on disease concepts. This pattern of documentation may reflect a difference in understanding and familiarity with $\mathrm{AD}$ among dermatologists and allergists/immunologists, suggesting that specialists have different approaches to how they understand and manage their patients' AD. Pediatric PCPs had relatively little documentation of broader disease issues (2.5\% of patients) but were comparable to PCPs with adult patients in terms of symptomatology-related notes.

\section{Limitations}

The first limitation of this study is that although the medications considered are AD-relevant, there is some overlap in use with other dermatology, asthma, and/or allergy conditions which may influence prescribing habits and potentially have an impact on the data from the studied notes. The second limitation of this study is that the overall generalizability of this study may be limited as the distribution of patients is more reflective of where small and single-provider practices are located than it is of population density. A third limitation of this study is that most practices were small and independent, and the effect of practice size and affiliation on management and treatment patterns was not considered. Additionally, provider notes reflect information deemed meaningful or important from the provider's point of view, thus note quality and content may be influenced by providers' familiarity with their patients and whether notes are written immediately following the visit or at some later point in time, a point not investigated in this study.

\section{CONCLUSION}

There are over 60 evaluation or assessment tools available for describing AD and AD severity [24]. They are not typically used in ambulatory practice, being primarily intended for clinical research. Investigators using real-world data are thus at a disadvantage in understanding the characteristics of patients' AD. This study supports the use of unstructured observational notes to provide that information. Providers across all specialties are documenting symptoms (primarily itch) and treatment. The bigram analyses of "itch", "atopic", "topical", and "systemic" reveal a substantial amount of information that can be leveraged through NLP 
processes to provide insights into current symptomatology and possibly severity and flare occurrence in a large and diverse ambulatory patient population. There is less documentation of the impact of $\mathrm{AD}$ on patient's life, possibly because of a lack of time to explore these issues during the typical office visit. Discussion of the role of AD on a patient's life could better inform physicians regarding management and treatment options that could ultimately lead to a better management of $\mathrm{AD}$.

\section{ACKNOWLEDGEMENTS}

Funding. Funding for this research and the journal's Rapid Service Fees was provided entirely by Eli Lilly and Company.

Authorship. All named authors meet the International Committee of Medical Journal Editors (ICMJE) criteria for authorship for this article, take responsibility for the integrity of the work as a whole, and have given their approval for this version to be published.

Authorship Contributions. All authors made significant contributions to the design of the research, interpretation of results, and development of the manuscript. Bogdanov, Lavalle, Sudaria, and Vasey were additionally responsible for the analysis of the data. The first draft of the manuscript was written by Vasey and all authors provided editorial input on it and on subsequent revisions. All authors have read and approved the final manuscript.

Disclosures. Evangeline J. Pierce is an employee of Eli Lilly and Company. Natalie N. Boytsov, Orin M. Goldblum, and Xiong Liu were employees of Eli Lilly and Company Health at the time this research was conducted. Joe J. Vasey, Kevin W. Lavelle, Theresa C. Sudaria and Alina N. Bogdanov are employees of Veradigm Health. Veradigm received funding from Eli Lilly and Company to conduct this study.
Compliance with Ethics Guidelines. The retrospective electronic health records dataset used for this study is HIPAA compliant and statistically certified for retrospective research without IRB review.

Data Availability. The datasets generated during and/or analyzed during the current study are not publicly available due to underlying data use agreements with the contributing data entities but are available from the corresponding author on reasonable request.

Open Access. This article is licensed under a Creative Commons Attribution-NonCommercial 4.0 International License, which permits any non-commercial use, sharing, adaptation, distribution and reproduction in any medium or format, as long as you give appropriate credit to the original author(s) and the source, provide a link to the Creative Commons licence, and indicate if changes were made. The images or other third party material in this article are included in the article's Creative Commons licence, unless indicated otherwise in a credit line to the material. If material is not included in the article's Creative Commons licence and your intended use is not permitted by statutory regulation or exceeds the permitted use, you will need to obtain permission directly from the copyright holder. To view a copy of this licence, visit http://creativecommons.org/licenses/by$\mathrm{nc} / 4.0 /$.

\section{REFERENCES}

1. Bieber T. Atopic dermatitis. $\mathrm{N}$ Engl J Med. 2008;358(14):1483-94. https://doi.org/10.1056/ NEJMra074081.

2. Berke S, Singh A, Guralnick M. Atopic dermatitis: an overview. Am Fam Physician. 2012;86(1):35-42.

3. Silverberg J. Public health burden and epidemiology of atopic dermatitis. Dermatol Clin. 2017;35(3): 283-9.

4. Lewis-Jones S. Quality of life and childhood atopic dermatitis: the misery of living with childhood eczema. Int J Clin Pract. 2006;60(8):984-92. 
5. Lifschitz C. The impact of atopic dermatitis on quality of life. Ann Nutr Metab. 2005;66:34-40.

6. Silverberg J, Gelfand J, Simpson E, Fuxench Z. Patient burden and quality of life in atopic dermatitis in US adults. Ann Allergy Asthma Immunol. 2018;121(3):340-7. https://doi.org/10.1016/j.anai. 2018.07.006.

7. Grob J, Revuz J, Ortonne J, Auquier P, Lorette G. Comparative study of the impact of chronic urticaria, psoriasis and atopic dermatitis on the quality of life. Br J Dermatol. 2005;152(2):289-95. https:// doi.org/10.1111/j.1365-2133.2005.06385.x.

8. Brenninkmeijer E, Schram M, Leeflang M, Bos J, Spuls P. Diagnostic criteria for atopic dermatitis: a systematic review. Br J Dermatol. 2008;158:754-65. https://doi.org/10.1111/j.1365-2133.2007.08412.x.

9. Nakamura T, Haider S, Colicino S, et al. Different definitions of atopic dermatitis: impact on prevalence estimates and associated risk factors. $\mathrm{Br} \mathrm{J}$ Dermatol. 2019;181:1272-9.

10. Schmitt J, Apfelbacher C, Spuls P. The Harmonizing Outcome Measures for Eczema (HOME) roadmap: a methodological framewaork to develop core sets of outcome measurements in dermatology. J Invest Dermatol. 2015;135:24-30.

11. Gustafson E, Pacheco J, Wehbe F, Silverberg J, Thompson W. A machine learning algorithm for identifying atopic dermatitis in adults from electronic health records. IEEE Int Conf Health Inf. 2017. https://doi.org/10.1109/ICHI.2017.31.

12. IQVIA. Physician office usage of electronic health records software: a market insights report. 2018. https://www.iqvia.com/locations/united-states/ library/fact-sheets/iqvia-market-insight-report-ehrsoftware-adoption. Accessed 28 May 2020.

13. Centers for Disease Control and Prevention National Center for Health Statistics. National ambulatory medical care survey: 2014 state and national summary tables. 2020. https://www.cdc. gov/nchs/data/ahcd/namcs_summary/2014_ namcs_web_tables.pdf. Accessed 28 May 2020.

14. Association of American Medical Colleges. Physician specialty data report. 2020. https://www.aamc. org/data-reports/workforce/interactive-data/ number-people-active-physician-specialty-2017. Accessed 26 June 2020.

15. Correale C, Walker C, Murphy L, Craig T. Atopic dermatitis: a review of diagnosis and treatment. Am Fam Physician. 1999;60(4):1191-8.

16. Silverberg J, Gelfand J, Margolis D, Fuxench Z, Simpson E, Ong P. Pain is a common and burdensome symptom of atopic dermatitis in United States adults. J Allergy Clin Immunol. 2019;7(8): 2699-706.

17. Vakharia P, Chopra R, Sacotte R, et al. Burden of skin pain in atopic dermatitis. Ann Allergy Asthma Immunol. 2018;119(6):548-52.

18. Chang Y, Chiang B. Sleep disorders and atopic dermatitis: a 2-way street? J Allergy Clin Immunol. 2018;142:1033-40.

19. Hon K, Leung T, Wong K, Chow C, Chuh A, Ng P. Does age or gender influence quality of life in children with atopic dermatitis? Clin Exp Dermatol. 2008;33:705-9.

20. Blome C, Radtke M, Eissing L, Augustin M. Quality of life in patients with atopic dermatitis: disease burden, measurement, and treatment benefit. Am J Clin Dermatol. 2016;17(2):163-9. https://doi.org/ 10.1007/s40257-015-0171-3.

21. Kiebert G, Sorensen S, Revicki D, et al. Atopic dermatitis is associated with a decrement in healthrelated quality of life. Int J Dermatol. 2002;41(3): $151-8$.

22. Lundberg L, Johannesson M, Silverdahl M, Hermansson C, Lindberg M. Health-related quality of life in patients with psoriasis and atopic dermatitis measured with SF-36, DLQI and a subjective measure of disease activity. Acta Derm Venereol. 2000;80:430-4.

23. Chernyshov P. Gender differences in health-related and family quality of life in young children with atopic dermatitis. Int J Dermatol. 2012;51(3):290-4.

24. Chopra R, Silverberg J. Assessing the severity of atopic dermatitis in clinical trials. Clin Dermatol. 2018;36(5):606-15. 